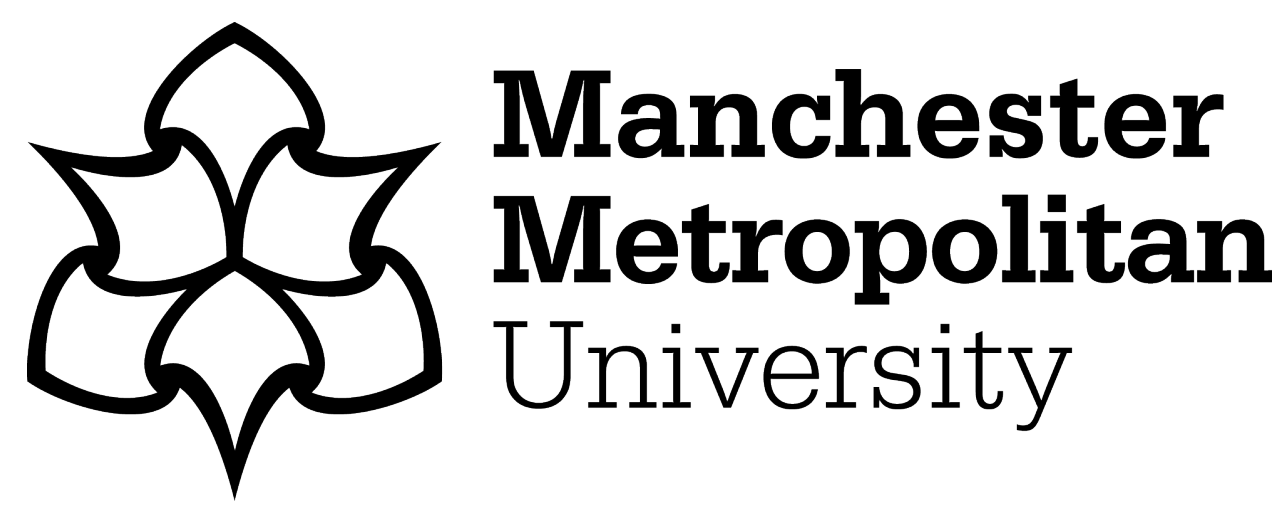

Naderi, $\mathrm{A}, \mathrm{Moen}, \mathrm{MH}$ and Degens, $\mathrm{H}$ ORCID logoORCID: https://orcid.org/0000-0001-7399-4841 (2020) Is high soleus muscle activity during the stance phase of the running cycle a potential risk factor for the development of medial tibial stress syndrome? A prospective study. Journal of Sports Sciences, 38 (20). pp. 2350-2358. ISSN 0264-0414

Downloaded from: https://e-space.mmu.ac.uk/626250/

Version: Accepted Version

Publisher: Taylor \& Francis

DOI: https://doi.org/10.1080/02640414.2020.1785186

Please cite the published version 


\title{
Is high soleus muscle activity during the stance phase of the running cycle a potential risk factor for the development of medial tibial stress syndrome? A prospective study
}

\author{
Aynollah Naderi, Maarten H. Moen and Hans Degens
}

\begin{abstract}
QUERY SHEET
This page lists questions we have about your paper. The numbers displayed at left are hyperlinked to the location of the query in your paper.

The title and author names are listed on this sheet as they will be published, both on your paper and on the Table of Contents. Please review and ensure the information is correct and advise us if any changes need to be made. In addition, please review your paper as a whole for typographical and essential corrections.

Your PDF proof has been enabled so that you can comment on the proof directly using Adobe Acrobat. For further information on marking corrections using Acrobat, please visit http://journalauthors.tandf.co.uk/production/acrobat.asp; https://authorservices.taylorandfrancis.com/how-to-correct-proofs-with-adobe/

The CrossRef database (www.crossref.org/) has been used to validate the references. Changes resulting from mismatches are tracked in red font.
\end{abstract}

\section{AUTHOR QUERIES}

Q1 Please note that the ORCID for Aynollah Naderi has been created from information provided through Manuscript. Please correct if this is inaccurate.

Q2 The ORCID for Maarten H Moen provided has been taken from Manuscript and verified against public API, and was found to be invalid. Please supply correct ORCID.

Q3 Please note that the ORCID for Hans Degens has been created from information provided through Manuscript. Please correct if this is inaccurate.

Q4 Please provide missing City for the affiliation $\mathrm{g}$.

Q5 Please provide missing Department for the affiliations $\mathrm{b}$ and $\mathrm{g}$.

Q6 The abstract is currently too long. Please edit the abstract down to no more than 200 words.

Q7 Please check whether the head levels has been set correctly.

Q8 If "Naderi et al., In press" been published, please provide the year for this citation.

Q9 If "Naderi et al., In press" been published, please give details for references list following journal style.

Q10 Please provide missing DOI or URL, if available, for the "Saxena et al., 1990" references list entry. 


\title{
Is high soleus muscle activity during the stance phase of the running cycle a potential risk factor for the development of medial tibial stress syndrome? A prospective study
}

\author{
Aynollah Naderi $\mathbb{D}^{\mathrm{a}}$, Maarten H. Moen $\mathbb{D}^{\mathrm{b}, \mathrm{c}, \mathrm{d}}$ and Hans Degens $\mathbb{( D}^{\mathrm{e}, \mathrm{f}, \mathrm{g}}$
}

aSchool of Sport Science, Shahrood University of Technology, Shahrood, Iran; 'bergman Clinics, Naarden, The Netherlands; 'The Sport Physician Group, OLVG West, Amsterdam, The Netherlands; 'Department of Elite Sports, National Olympic Committee and National Sports Federation, Arnhem, The Netherlands; 'School of Healthcare Science, Manchester Metropolitan University, Manchester, UK; fInstitute of Sport Science \& Innovations, Lithuanian Sports University, Kaunas, Lithuania; ${ }^{9}$ University of Medicine \& Pharmacy of Targu Mures, Rumania

ABSTRACT

The aim of this study was to assess the impact of lower leg muscle activity during the stance phase of running on the development of medial tibial stress syndrome (MTSS). In 123 healthy participants (18.2 \pm 0.8 years), dynamic and static foot posture, and soleus and tibialis anterior muscle activity during the stance phase of running were measured before a 17-week track-and field-course. After the course, MTSS was identified in $20.5 \%$ of the participants. MTSS participants have a higher body mass $(E S=1.13)$ and body mass index (BMI) $(E S=1.31)$, lower previous vigorous physical activity level $(E S=0.84)$ and $\mathrm{VO}_{2 \max }(\mathrm{ES}=0.61)$, greater dynamic foot pronation $(\mathrm{ES}=0.66)$, higher soleus peak EMG amplitude during the absorption $(E S=0.60)$ and propulsion phases $(E S=0.56)$ of running, and previous history of MTSS $(\mathrm{OR}=6.38)(p<0.05)$. Stepwise logistic regression showed BMI, dynamic foot index, soleus peak EMG amplitude during propulsion, history of MTSS and previous experience of vigorous physical activity were predictors of MTSS. The model predicted $96.6 \%$ of the healthy participants and $56.5 \%$ of the MTSS participants and correctly classified $88.4 \%$ of overall cases. Coaches and sports-medicine professionals that screen for injury risk should consider adopting a comprehensive evaluation that includes these parameters.

\section{ARTICLE HISTORY}

Accepted 11 June 2020

KEYWORDS

Shin splint; injury-prevention ; predicting injury; running; muscle activity; electromyography

\section{Introduction}

Medial tibial stress syndrome (MTSS) is defined as exerciseinduced pain along the posteromedial border of the distal twothirds of the tibia that occurs during or after activity and can be diagnosed by palpation of this area (Winters et al., 2018), where compartment syndrome or stress fracture is excluded. MTSS is a common overuse condition associated with the weightbearing activity (Winters et al., 2016). The prevalence of MTSS in runners is somewhere between $13.6 \%$ and $20 \%$ (Lopes et al., 2012) and accounts for up to $60 \%$ of lower limb injuries (Couture \& Karlson, 2002). MTSS may limit participation in sports and recreational activities. Moreover, MTSS has a high recurrence rate (Yates \& White, 2004) and is difficult to treat. Therefore, prevention of MTSS is crucial.

Although the characteristic signs and symptoms of MTSS are well established, there is disagreement in the literature regarding the aetiology of MTSS (Alfayez et al., 2017). There are two

40 prevailing pathophysiological theories for MTSS (Bouche \& Johnson, 2007; Tweed et al., 2008). The tibia fascia-traction theory (TFTT) suggests that muscles apply too much of a traction force on the tibia and its periosteum (Bouche \& Johnson, 2007), and the bone stress reaction theory (BSRT) suggests that bone remodelling to repetitive stress is inadequate (Tweed et al., 2008).

Lower leg muscles play a crucial role in controlling foot flexibility and rigidity during foot rollover (Murley et al., 2009), and are considered as a prerequisite to absorb forces and protect bones of the lower limb from excessive shock during weight-bearing activities (Naderi et al., in press). In addition, foot pronation is a well-known risk factor for MTSS (Becker et al., 2018; Newman et al., 2013) that results in a medial shift of the centre of pressure (COP) under the foot during the stance phase of running (Becker et al., 2018; Naderi et al., 2019). This increased pronation may cause a higher activity of tibialis anterior (TA) and soleus (SO) muscles to minimize excessive pronation (Hunt \& Smith, 2004; Murley et al., 2009). Despite the protective role of these muscles, their increased and longer duration of activity may increase the strain in the tibial fascia and/or disrupt bone remodelling through increased strain of the cortical bone (Milgrom et al., 2007).

In line with the above suggestion, it has been shown that increased TA muscle activity during manual muscle tests in people with MTSS causes a pain characteristic of MTSS (DeLacerda, 1980). Similarly, it has been shown that large and long duration of traction forces associated with over-activity of the SO muscle may also result in severe strains to the distal third of the posteromedial tibia (Beck \& Osternig, 1994; Brown, 2016; Stickley et al., 2009). If, without proper recovery, this is repeated over and over again during running it may ultimately lead to MTSS. However, these were retrospective studies in individuals with MTSS, and one can therefore not conclude as to the cause or effect of increased TA or SO activity on the 
75 development of MTSS. To evaluate whether increased TA or SOL activity indeed are risk factors for MTSS, prospective studies are needed (Becker et al., 2018). The purpose of the current study, therefore, was to compare prospectively TA and SO muscle activity during running between university runners who developed MTSS and those who did not. In addition, we sought to determine whether TA and SO muscle activity predicted which university runners developed MTSS. We hypothesized that compared to controls, university runners who developed MTSS demonstrate increased TA and SO muscle activity during running.

\section{Methods}

\section{Participants}

One hundred twenty-three healthy students (20-30 years old) were recruited in September - December 2016 ( $n=25)$,

90 January - May 2017 ( $\mathrm{n}=30$ ), September - December 2017 ( $n=33)$, and January - May $2018(n=35)$, and participated in our study after providing written informed consent. All participants were first-year bachelor's degree physical education students, who were active in running sports as the main course of their study. Their semester programme consists of four different sports courses such as running (sprint and distance), basketball, handball, and indoor soccer for approximately 12 hours per week. All procedures were approved by the Institutional Review Board of Shahrood University of Technology and were in accordance with the Declaration of Helsinki. Inclusion criteria were being between the age of 20 to 30 years and participation in $>90 \%$ of sessions of our study. Participants with a current or history of lower limb pain, ache or soreness within the last 6 months, a history of lower limb surgery, a congenital deformity of the lower limb and/or an obvious leg-length discrepancy were excluded. Therefore, all participants were free of lower limb musculoskeletal injuries (including MTSS) at the time of enrolling in our study, but they may have had this problem 6 months before.

\section{Procedure}

Data collection took place during 17 weeks in a research laboratory before the start of each of the respective athletic activities. All participants completed a questionnaire to obtain the age, limb dominance, running sport experience (number of sessions in a week and duration of running per week), and foot orthoses or tape use that was performed by a sport science specialist. Before participating in our study, participants underwent a medical examination performed in a clinical physiotherapy clinic by a sport physiotherapist. This examination also involved questions regarding the previous history of lower-extremity injury or pain, history of MTSS, and site of symptoms. In a face-to-face interview, the Iranian version of the international physical activity questionnaire (IPAQ) was used to assess the physical activity (PA) levels of each participant (Moghaddam et al., 2012). The total weekly number of PA minutes was calculated for the following activities: vigorous (heavy lifting, digging, aerobics, or fast bicycling), moderate (e.g., carrying light loads, bicycling at a regular pace, or doubles tennis), and walking within the domains of occupation, transportation, housework/gardening and leisure-related activities. Then, total reported duration engaged in each PA was multiplied by the corresponding value of metabolic equivalent (MET; i.e., the ratio of work metabolic rate to the standard resting metabolic rate) to calculate MET-h per week for each level of PA. In addition, VO2max was estimated from a submaximal 1-mile track jog that was conducted before the start of the academic course as follows:

$$
2 V_{2} O_{22 \max } 2=1002.5+82.344 *(2 S e x) 2-02.1636 *(2 B M)
$$

$$
2-12.438 *(2 \text { Time, } \min 2 . m i l e-1) 2-02.1928 *(2 H R) 2,
$$

where sex is 0 for women and 1 for men, BM is body mass in $\mathrm{kg}$, and HR is heart rate. This test has been shown to provide a valid measure of aerobic fitness of college-aged individuals (George et al., 1993). Anthropometric data such as height, mass and BMI were recorded. A digital scale (SECA 760, Vogel \& Halke GmbH $\&$ Co., Hamburg, Germany) was used to measure the body mass to the nearest $0.1 \mathrm{~kg}$ and height was measured to the nearest $0.1 \mathrm{~cm}$. The body mass index (BMI) was calculated as body mass.height ${ }^{-2}\left(\mathrm{~kg} \cdot \mathrm{m}^{-2}\right)$.

\section{Dynamic and static foot posture measurement}

Dynamic and static foot posture were evaluated using the dynamic arch index (DAI) and foot posture index (FPI) during running. The DAl is the ratio of the midfoot contact area relative to the whole footprint area excluding the toes (De Cock et al., 2006). For calculating this index, total footprint length, except the toe print is measured from the centre of the heel to the small toe's tip and is divided into three equal halves. The middle third area divided by the total area gives the arch index $\left(\mathrm{DAI}=\frac{\mathbf{B}}{\mathbf{A}+\mathbf{B}+\mathbf{C}} \times 100\right)$ (Figure 1). A high ratio indicates higher foot pronation; <21\% high arch, 21\% -26\% normal arch and $\geq 26 \%$ low arch (Cavanagh \& Rodgers, 1987). The dynamic pressure distribution during running trials was assessed using a force plate (RsScan International, Paal, Belgium, $40 \times 100 \mathrm{~cm}, 8192$ sensors, $253 \mathrm{~Hz}$ ) that was placed in the middle of a 12-m-long runway (Naderi et al., 2019). The RS scan system was calibrated according to the guidelines of the manufacturer before each session. The FPI is a clinical method to quantify the degree to which a foot is pronated or supinated. FPI consists of six criterion-based observations: (1) talar head palpation, (2) curvature at the lateral malleoli, (3) inversion/eversion of the calcaneus, (4) talonavicular bulging, (5) congruence of the medical longitudinal arch, and (6) abduction/adduction of the forefoot on the rare-foot. Each observation was scored on a 5-point Likert-type scale (from -2 for clear signs of supination to +2 for clear signs of pronation) that was summed to provide a quantification of foot posture in the 3 cardinal body planes. The final score ranged from -12 to +12 . FPI scores of -12 to -6 were considered highly supinated, -5 to -1 considered supinated, 0 to 5 considered normal, 6 to 9 considered pronated, and $>10$ considered highly pronated (Redmond et al., 2006). To eliminate inter-rater bias, the same investigator conducted all physical examinations. To minimise any order-bias of the 


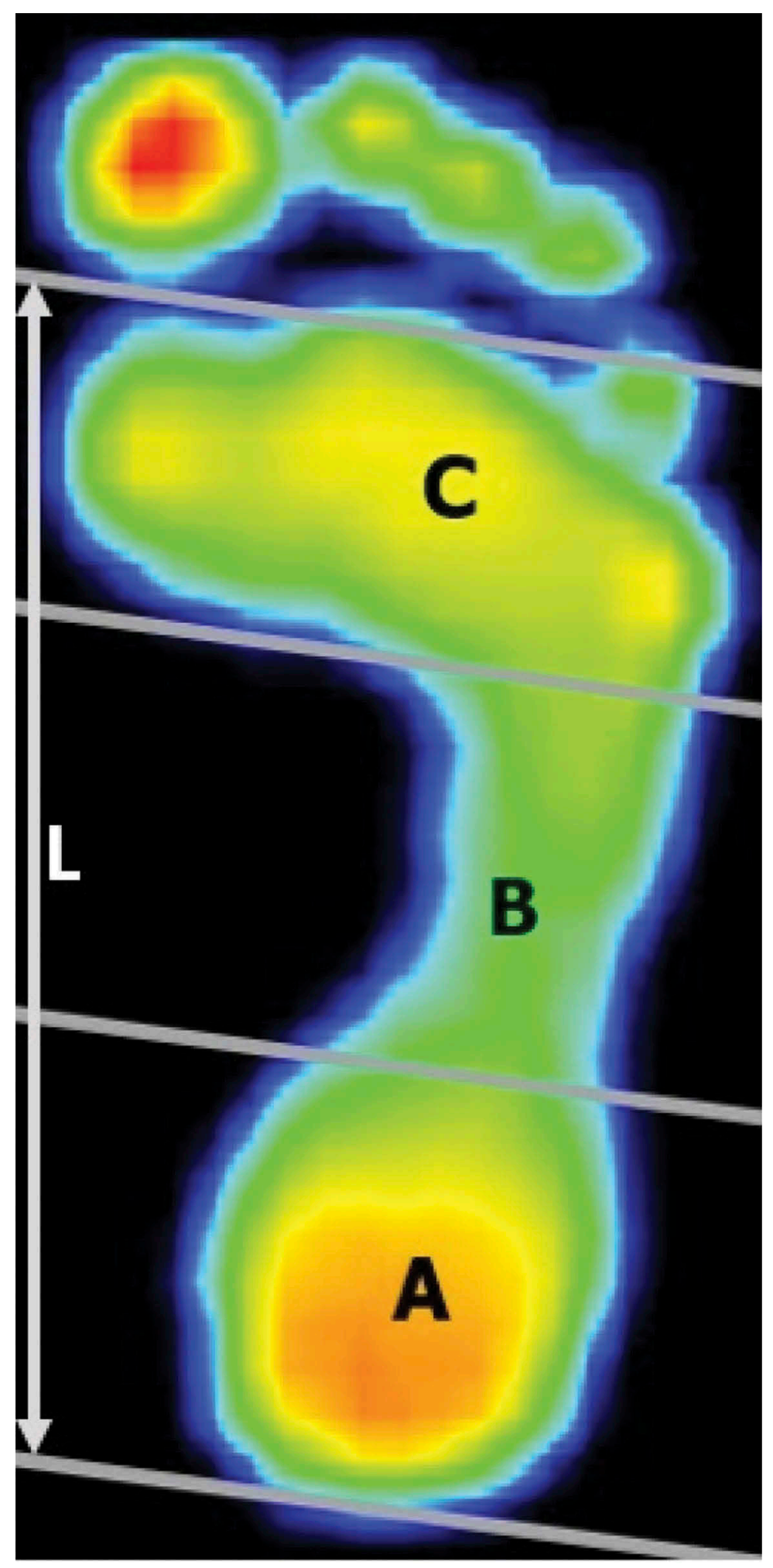

Figure 1. Dynamic arch index, $A$ - forefoot, $B$ - midfoot, $C$ - rearfoot, $L-$ length of the foot. Dynamic arch index $\left.=\frac{B}{A+B+C} \times 100\right)$.

three physical examinations, the order was randomised by the throw of a dice.

\section{EMG measurement}

185 Measurements of muscle activity were performed for both limbs during a single session at the start of the academicsemester (running course). An experienced certified sport science specialist conducted the EMG measurements of the tibialis anterior (TA) and soleus (SO) muscles. The EMG data were sampled using a portable EMG system (MT8 Telemetry system; MIE Medical Research Ltd., Leeds, UK) at a sample rate of $1000 \mathrm{~Hz}$, amplified with a gain 4000, bandwidth of 20-500 Hz, common mode rejection ratio of $>100 \mathrm{~dB}$. A pair of pre-gelled $\mathrm{Ag} / \mathrm{AgCl}$ surface electrodes (circular shape with 
$19510 \mathrm{~mm}$ diameter) were placed parallel to the muscle belly with $20 \mathrm{~mm}$ inter-electrode distance. To archive acceptable impedance level before placement of the electrodes, the skin overlying the corresponding muscle bellies was gently shaved, abraded, and cleaned with alcohol (70\% Ethanol- $\left.\mathrm{C}_{2} \mathrm{H}_{5} \mathrm{OH}\right)$ according to the SENIAM recommendations (Table 1). The cables and amplifiers were taped to the skin to minimize movement artefacts. A single electrode (ground electrode) was placed over the patella. All clinical assessments took place at sport rehabilitation lab of Shahrood University of Technology.

A maximum voluntary isometric contractions (MVIC) against a fixed load, comprised of a gradual and continuous 2-s buildup followed by a maximum 4-s effort, were used to obtain the maximal EMG. The maximal EMG was measured in a $600-\mathrm{ms}$ window in the middle of the plateau and the average root means square (RMS). Three consecutive maximum efforts separated by a 2 min recovery period were undertaken and the largest RMS of the three trials was used as the maximal EMG.

After assessing the maximal EMG, the participant performed a number of practice trials to become familiar with the test procedures. To control for the effects of running speed on muscle activity (Kyröläinen et al., 2005), the participants ran at a speed of $6 \mathrm{mph} \pm 5 \%$ over a $15-\mathrm{m}$ indoor runway, monitored by two sets of infrared photocells. Once the participants indicated they were comfortable with the procedures and the EMG device, EMG data were also collected during running. The RMS of all EMG data were normalised to the RMS of the EMG signal during an MVIC.

Participants completed three running trials and to ensure the running velocity was constant, only the third or fourth stride of each trial, depending on the quality of the footswitch signal, was chosen for analysis. Circular force-sensitive resistors (footswitch) with a 13-mm diameter (Foot Switch, Kissei Comtec, Nagano, Japan) were used to measure the temporal characteristics of the running cycle, defined as one heel strike to the next one with the same foot. The resistors were placed on the plantar surface of the interphalangeal joint of the hallux and the most posterior plantar aspect of the calcaneus to record the timing of heel contact, toe contact, heel off and toe-off (Scott et al., 2012). According to (Lohman lii et al., 2011), the running cycle was divided into a stance (when the footswitches had contact with the ground) and a swing (when the footswitches did not have contact with the ground) phase. The stance phase of the running cycle was subdivided into absorption and propulsion phases. The period between first ground contact of the footswitch under the heel until the end of ground contact was defined as the absorption phase. The propulsion phase was defined as the period between the loss of ground contact of the footswitches under the heel till the footswitches under the hallux leave the ground (Lohman lii et al., 2011).

Raw EMG data from the MVICs and running trials were smoothed using a 50-ms moving window RMS algorithm. EMG data were quantified as the RMS values using MYO-DAT

Table 1. Location of EMG electrodes according to the SENIAM recommendations on the tibialis anterior (TA) and soleus (SO) muscles.

approximately $1 / 3$ of the distance from tip of the fibula to the tip of the
medial malleolus

5.0 EMG analysis software ${ }^{\circledR}$ (MIE Medical Research Ltd, Leeds, UK) according to the following equation:

$$
R M S \text { value }[\mathbf{I}]=\sqrt{\frac{\sum_{i=n}^{n+N-1} \mid \text { Data }\left._{\text {Raw }}[\mathbf{i}]\right|^{2}}{\mathbf{N}}}
$$

where $\mathrm{I}=$ index of RMS data; $\mathrm{i}=$ index of raw data; $\mathrm{N}=$ number of data points in RMS calculation; and $\mathrm{n}=[1$, $\mathrm{N}+1,2 \mathrm{~N}+1, \ldots]$. For example, our data sampling frequency was $1000 \mathrm{~Hz}$, and with an averaged 50-ms data period, then $\mathrm{N}=20$. EMG normalization was performed by dividing each EMG data point to the EMG data during the MVIC of the corresponding muscle. EMG data were time-interpolated over a time base with 101 points for individual running cycles.

\section{Follow-up}

After the initial data collection, the participants were followed for 17 weeks. The amount of extramural (physical activities beyond sports lessons at the university) and non-supervised physical activities were registered daily in an activity diary (pre-printed form), and included the type and duration of the activity. Participants were asked to report any tibial pain to the class athletic trainer. If they reported any tibial pain, they were directed to the sport physician to diagnose the presence or absence of MTSS. For this study, MTSS was defined as pain experienced along the posteromedial border of the tibia during exercise, and not pain caused by ischaemic disorders or stress fractures (Yates \& White, 2004). Participants were classified with MTSS if they fulfilled the following criteria and the absence of other symptoms of exercise-induced leg pain (Edwards et al., 2005; Yates \& White, 2004):

- Pain was induced by exercise and lasted for hours or days after exercise

- Pain was located in the distal half of the posteromedial tibia and covered an area with a length of more than $5 \mathrm{~cm}$

- Palpation of the tibial posteromedial border induced diffuse discomfort that was restricted to this area (Naderi et al., 2019)

Participants were considered free from MTSS if any signs of cramp, burning pain, paraesthesia or compression in the lower leg, characteristic of chronic compartment syndrome, were present.

Based on the reporting of pain and the diagnosis by a sport physiotherapist with 12 years' experience, participants were divided into two groups: an MTSS group (23 students who developed MTSS) and a CON group (89 students who did not have any lower-extremity overuse injury). For students that developed unilateral MTSS, only the painful lower-extremity data were used. For bilateral MTSS, the most painful side (as determined by visual analog scale) was used in the statistical analysis. The percentage of non-dominant/dominant legs in the CON group was matched with the percentage of non-dominant /dominant legs in the MTSS group by creating a similar ratio among the participants in the CON group and MTSS group.

\section{Data analysis}

SPSS statistical software (version 18.0, SPSS Inc., Chicago, IL, USA) was used to perform all statistical analyses. The Shapiro- 
Wilk test was used to assess the normality of continuous variables. MTSS participants were compared with CON participants using independent t-tests for continuous dependent variables and $x^{2}$ tests for discrete dependent variables. The effect size of Cohen's d (ES) was calculated for all continuous variables and Odds Ratios (ORs) for discrete dependent variables. Then, to avoid multicollinearity a bivariate correlation was conducted among dependent variables prior to performing regression analysis. If two or more variables correlated highly $(r>0.6)$, one of the variables was removed from the model. Then, a multivariate analysis was performed using forward stepwise conditional logistic regression to determine the major risk factors for MTSS. Variables were excluded from the analysis if the correlation with MTSS risk had a $p \geq 0.10$. The ORs with $95 \%$ Confidence Intervals (Cls) of these variables were calculated. The Hosmer-Lemeshow goodness-of-fit test was used to evaluate the model's fit. The significance level was set a priori at $p<0.05$.

\section{Results}

Of the initial 123 participants that completed the survey and physical examination, 112 (92.5\%) participants were included in the data analysis (Table 2). During the 17-week follow-up period, 23 students (20.5\%) developed MTSS. Eleven (8.9\%) developed other lower-extremity injuries and were excluded from the comparison. Two (1.6\%) participants in the MTSS group and nine $(7.3 \%)$ in the CON group suffered from overuse injuries such as Achilles tendinopathy and plantar fasciitis, and acute injuries such as adductor and hamstring strains or ankle sprains (Table 2).
As shown in Table 2, there was no significant difference in the incidence of MTSS between women (incidence rate $=22.95 \%)$ and men (incidence rate $=17.65 \%)\left(x^{2}=1.4\right.$, $p<0.24)$. There were also no significant differences between the CON and MTSS group concerning age and height $(p>0.05)$, but the body mass $(p<0.001, \mathrm{ES}=1.13)$ and BMI $(p<0.001$, $\mathrm{ES}=1.31$ ) were higher in the MTSS than CON group. The $\mathrm{VO}_{2 \max }$ of MTSS participants was lower than that of the CON men and women $(p<0.01, \mathrm{ES}=0.61)$. The MTSS group had also less vigorous physical activity $(p<0.001, \mathrm{ES}=0.84)$ than the CON group, but there was no significant difference between groups for walking and moderate physical activity ( $p>0.05$; Table 2 ). Mean DAI score in the MTSS participants was higher than that of the CON group $(p<0.01$, ES $=0.66)$, but there was no significant difference between groups in the FPI score $(p>0.05)$. The MTSS group was more likely to have a history of MTSS $(p<0.001)$ and to use foot orthoses $(p<0.04)$ than the CON group participants, but there was no significant difference between groups in lower-extremity injury $(p>0.10)$ and supplements use ( $p=0.054$ ) (Table 2).

Comparisons of EMG variables between the MTSS and CON groups are presented in Figure 2. No significant differences in time of peak RMS $(t=1.1, p=0.27 ; \mathrm{ES}=0.30$ and $t=0.27$, $p=0.78$; ES $=0.12)$, peak RMS $(t=1.7, p=0.1 ; \mathrm{ES}=0.39$ and $t=1.8, p=0.1 ; \mathrm{ES}=0.43)$, and mean RMS $(t=1.4, p=0.2$; $\mathrm{ES}=0.33$ and $t=1.8, p=0.1 ; \mathrm{ES}=0.42$ ) of the EMG were detected for the TA during the absorption and propulsion of running, respectively. For SO, the peak RMS of the EMG, however, was higher in the MTSS than the CON group during the absorption $(t=2.6, p=0.01 ; \mathrm{ES}=0.60)$ and propulsion $(t=2.4, p=0.02$; $\mathrm{ES}=0.56)$ phase of running. However, the time of peak $(t=0.40$,

Table 2. Participant characteristics.

\begin{tabular}{|c|c|c|c|c|c|c|c|c|}
\hline \multicolumn{3}{|l|}{ Variables } & $\begin{array}{c}\text { MTSS } \\
(n=23)\end{array}$ & $\begin{array}{c}\mathrm{CON} \\
(\mathrm{n}=89)\end{array}$ & Mean inter-group difference $(95 \% \mathrm{Cl})$ & $t / X^{2} a$ & $\mathrm{p}$-value & $\begin{array}{c}\text { Effect size } \\
\text { (Cohen's d/Odds ratio) }^{b}\end{array}$ \\
\hline \multicolumn{3}{|c|}{ Sex (male/female) } & $9 / 14$ & $42 / 47$ & & $\mathrm{t}=1.4$ & 0.24 & $\mathrm{OR}=0.72$ \\
\hline \multicolumn{3}{|l|}{ Age $(y)$} & $23.1 \pm 2.2$ & $22.9 \pm 2.1$ & $0.3(-0.7$ to 1.3$)$ & $t=0.3$ & 0.75 & $d=0.14$ \\
\hline \multicolumn{3}{|l|}{ Mass $(\mathrm{kg})$} & $72.0 \pm 8.6$ & $64.6 \pm 7.3$ & 7.3 (3.8 to 10.9$)$ & $\mathrm{t}=4.1$ & 0.001 & $d=1.13$ \\
\hline \multicolumn{3}{|l|}{ Height $(\mathrm{cm})$} & $172 \pm 4$ & $174 \pm 6$ & $-2(-2.9$ to 2.4$)$ & $\mathrm{t}=0.5$ & 0.64 & $d=0.34$ \\
\hline \multicolumn{3}{|c|}{ Body mass index $\left(\mathrm{kg} \cdot \mathrm{m}^{-2}\right)$} & $21.5 \pm 2.3$ & $24.0 \pm 3.0$ & 2.5 (1.4 to 3.7$)$ & $\mathrm{t}=4.4$ & 0.001 & $d=1.31$ \\
\hline \multicolumn{3}{|c|}{$\mathrm{VO}_{2 \max }\left(\mathrm{mL} \mathrm{kg}^{-1} \cdot \mathrm{min}^{-1}\right)$} & $44.6 \pm 5.0$ & $47.9 \pm 5.7$ & $-3.4(-6.0$ to -0.8$)$ & $t=2.59$ & 0.01 & $d=0.61$ \\
\hline \multicolumn{3}{|c|}{ Walking (METs-h/week) } & $21.6 \pm 2.9$ & $22.8 \pm 2.6$ & $-1.2(-2.5$ to 0.01$)$ & $t=1.9$ & 0.08 & $d=0.44$ \\
\hline \multicolumn{3}{|c|}{ Moderate PA (METs-h/week) } & $17.1 \pm 4.9$ & $16.9 \pm 5.0$ & $0.22(-2.1$ to 2.5$)$ & $t=0.19$ & 0.9 & $d=0.05$ \\
\hline \multicolumn{3}{|c|}{ Vigorous PA (METs-h/week) } & $17.1 \pm 6.7$ & $23.5 \pm 6.7$ & $-6.5(-9.6$ to -3.4$)$ & $\mathrm{t}=3.9$ & 0.001 & $d=0.84$ \\
\hline \multicolumn{3}{|c|}{ Previous sport activities per week (min) } & $82.4 \pm 17.4$ & $103.1 \pm 19.2$ & $-20.6(-29.4$ to -11.9$)$ & $\mathrm{t}=4.7$ & 0.001 & $d=1.04$ \\
\hline \multirow[t]{4}{*}{ DAI (\%) } & Mean \pm SD & & $0.27 \pm 0.05$ & $0.24 \pm 0.04$ & 0.03 (0.01 to 0.05 ) & $t=2.6$ & 0.01 & $d=0.66$ \\
\hline & Count n (\%) & Low & $10(43.5)$ & $16(18)$ & - & $X^{2}=8.2$ & 0.02 & - \\
\hline & & Normal & $10(43.5)$ & $66(74)$ & - & & & \\
\hline & & High & $3(13)$ & $7(8)$ & - & & & \\
\hline \multirow{4}{*}{ FPI (score) } & Mean \pm SD & & $3.5 \pm 3.7$ & $2.6 \pm 2.8$ & $0.9(-0.8$ to 2.6$)$ & $\mathrm{t}=0.8$ & 0.4 & $d=0.30$ \\
\hline & Count n (\%) & Pronate & 6 & 14 & - & $X^{2}=2.4$ & 0.3 & - \\
\hline & & Normal & 14 & 68 & - & & & \\
\hline & & Supinate & 3 & 7 & - & & & \\
\hline \multicolumn{3}{|c|}{ Previous history of LL injury,(n) (Yes/No) } & $8 / 15$ & $17 / 72$ & - & $X^{2}=2.6$ & 0.15 & $\mathrm{OR}=2.26$ \\
\hline \multicolumn{3}{|c|}{ Previous history of MTSS, (n) (Yes/No) } & $12 / 11$ & $13 / 76$ & - & $X^{2}=14.8$ & 0.001 & $\mathrm{OR}=6.38$ \\
\hline \multicolumn{3}{|c|}{ Foot orthoses using, (n) (Yes/No) } & $11 / 12$ & $21 / 68$ & - & $X^{2}=5.3$ & 0.04 & $\mathrm{OR}=2.69$ \\
\hline \multicolumn{3}{|c|}{ Supplements intakes, (n) (Yes/No) } & $6 / 17$ & $22 / 67$ & - & $X^{2}=0.02$ & 0.54 & $\mathrm{OR}=1.07$ \\
\hline \multirow{2}{*}{\multicolumn{3}{|c|}{ Side affected, (n) (dom/nondom/bilateral) }} & $11 / 5 / 7$ & - & - & - & - & - \\
\hline \multicolumn{2}{|c|}{ Target side, $(\mathrm{n})(\%)($ dom/nondom) } & & $14 / 9$ & $54 / 35$ & - & - & - & - \\
\hline \multicolumn{3}{|c|}{ Pain intensity (VAS pain $0-10$ ) } & $62.1 \pm 8.7$ & - & - & - & - & - \\
\hline
\end{tabular}

Abbreviations: MTSS; Medial Tibial Stress Syndrome, CON; Control Group, PA; Physical Activity, MET; Metabolic Equivalent, DAl; Dynamic Arch Index, FPI; Foot Posture Index, LL; Lower limb. 95\% Cl; 95\% Confidence Interval.

${ }^{a} t$, the test statistic for the independent $t$-tests; $x^{2}$, the test statistic for the Chi-squared tests. ${ }^{b}$ Cohen's $d$ effect size for continuous variables; OR, effect size for discrete variables. 

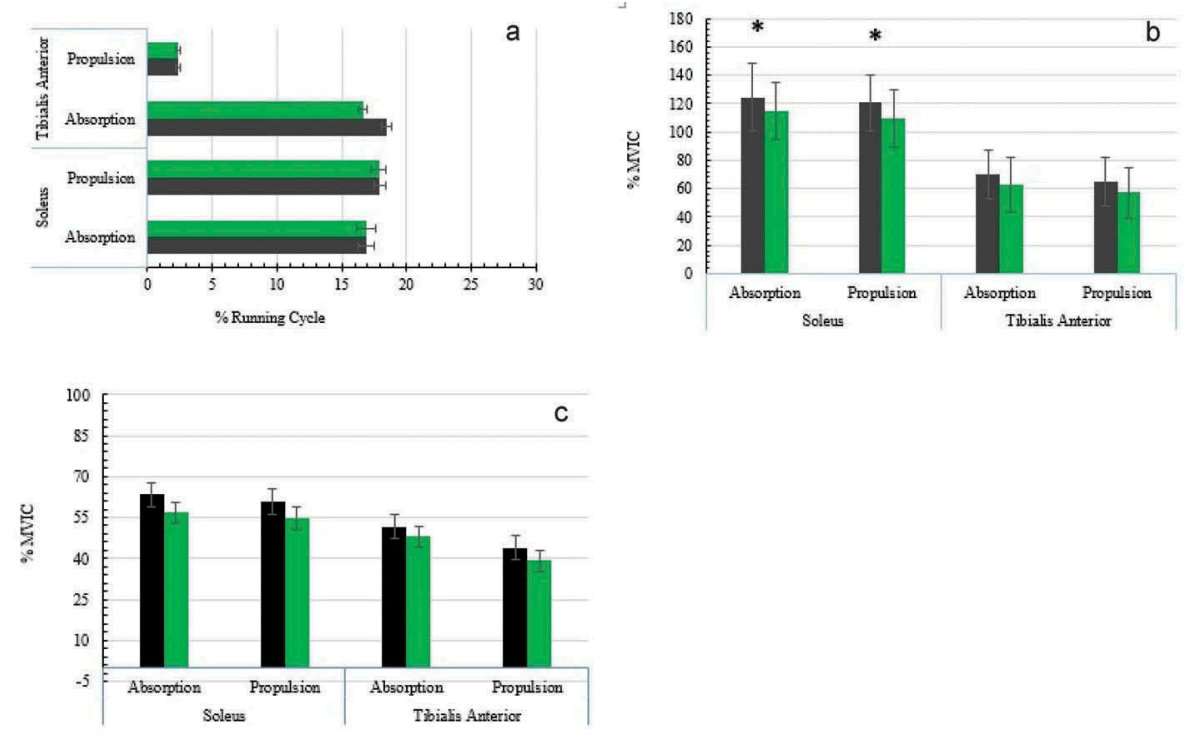

Figure 2. Comparison of time to peak (Mean+SD)(a), Peak RMS (b), and Mean RMS (c) of TA and SO muscles for who developed medial tibial stress syndrome and participants during stance phases of running. Abbreviation: MTSS; Medial Tibial Stress Syndrome, CON; Control Group, MVIC; Maximum Voluntary Isometric Contractions, SO; soleus, and TA; tibialis anterior. *Significant difference at $p<0.05$.

$p=0.8 ; \mathrm{ES}=0.1$ and $t=0.67, p=0.5 ; \mathrm{ES}=0.11)$ and mean RMS $(t=1.8, p=0.09 ; \mathrm{ES}=0.46$ and $t=1.6, p=0.1 ; \mathrm{ES}=0.43$ ) of $\mathrm{EMG}$ for this muscle were not significantly different during the absorption and propulsion of running, respectively (Figure 2).

Bivariate correlations revealed in the TA muscle a strong correlation between the peak with the mean RMS during the absorption $(r=0.78, p<0.001)$ and propulsion phase of running $(r=0.71, p<0.001)$. Also, in the SO, there was a strong correlation between the peak and mean RMS during the absorption $(r=0.70, p<0.001)$ and propulsion phase of running $(r=0.62$, $p<0.001)$. Body mass correlated highly with BMI $(r=0.86$, $p<0.001$ ). Based on these observations, BMI, peak RMS of TA and $\mathrm{SO}$ during the absorption and propulsion phase of running were retained for input into the regression model. Bivariate correlation coefficients between all other variables fed into the logistic regression were ranged from.021 to. 41 .

The results of multivariate stepwise logistic regression are found in Table 3. The Hosmer-Lemeshow test showed a good fit to the model $\left(x^{2}(8)=6.24, p=0.62\right)$. The final stepwise logistic regression model was statistically significant $\left(x^{2}(1,5)=5.36\right.$; $p<0.02$ ) and explained $54.7 \%$ (Nagelkerke $R^{2}$ ) of the variance

Table 3. Stepwise multivariate logistic regression analyses.

\begin{tabular}{lccccc}
\hline Variables & $\mathrm{B}$ & $\mathrm{SE}$ & Wald & $\mathrm{p}$-value & OR (95\% Cl) \\
\hline BMI $\left(\mathrm{kg} \cdot \mathrm{m}^{-2}\right)$ & 0.62 & 0.16 & 15.5 & 0.001 & $\begin{array}{c}1.85(1.36 \text { to } \\
2.52)\end{array}$ \\
Vigorous PA (METs-h/week) & -0.28 & 0.13 & 4.70 & 0.03 & $\begin{array}{c}0.75(0.58 \text { to } \\
0.97)\end{array}$ \\
DAI (\%) & -0.05 & 0.02 & 5.92 & 0.02 & $\begin{array}{c}1.15(1.02 \text { to } \\
1.28)\end{array}$ \\
$\begin{array}{c}\text { Previous history of MTSS, (n) } \\
\text { (Yes/No) }\end{array}$ & 1.54 & 0.67 & 5.27 & 0.02 & $\begin{array}{c}4.67(1.25 \text { to } \\
17.37)\end{array}$ \\
$\begin{array}{c}\text { Peak RMS of SO during } \\
\text { propulsion (\% MVIC) }\end{array}$ & 0.07 & 0.03 & 6.97 & 0.01 & $\begin{array}{c}1.05(1.01 \text { to } \\
1.08)\end{array}$ \\
\hline
\end{tabular}

Abbreviations: B; Regression Coefficient, SE; Standard Error, OR; Odds Ratio, 95\% $\mathrm{Cl} ; 95 \%$ Confidence Interval, BMl; Body Mass Index, PA; Physical Activity, MET; Metabolic Equivalent, DAl; Dynamic Arch Index, MTSS; Medial Tibial Stress Syndrome, SO; Soleus, RMS; Root Mean Square, MVIC; Maximum Voluntary Isometric Contractions. in susceptibility to MTSS and correctly classified $88.4 \%$ of cases ( $96.6 \%$ of healthy individuals and $56.5 \%$ of MTSS individuals). In the final model, greater BMI and dynamic foot index were respectively associated with an increase of $85 \%$ and $15 \%$ of the likelihood of MTSS occurrence and previous history of MTSS increased the risk of MTSS 4.67 times. In addition, previous experience of vigorous PA was associated with a $25 \%$ decrease of the likelihood of developing MTSS and greater peak RMS of SO during propulsion increased the risk of MTSS by $5 \%$.

\section{Discussion}

The purpose of the current study was to prospectively compare the activity of the TA and SO muscles during running between university runners who developed MTSS and those who did not. The results of our study showed that participants who developed MTSS had a significantly higher SO peak EMG RMS during the absorption and propulsion phase of running. The body mass and BMI were larger, previous vigorous physical activity was less, and dynamic arch index during running was lower in those who developed MTSS comparison to the participants who did not develop MTSS. In addition, a previous history of MTSS and the use of foot orthoses were also more prevalent in those that developed MTSS. Of the variables used in a multivariate model, the BMI, previous history of MTSS, DAI during running, previous experience of vigorous PA, and Peak RMS of SO during propulsion correctly predicted $88.6 \%$ of the participants who eventually developed MTSS. These results may indicate that those variables are important when screening individuals for the risk of developing MTSS.

\section{$B M I$ and body mass}

In the present study, the body mass and BMI were higher in the MTSS participants than participants of the CON group. Odds of developing MTSS were 1.85 times higher in people with a high 
410 BMI, which is in line with previous studies (Newman et al., 2013; Reinking et al., 2010). Possibly, increased bending of the tibia and pronounced microdamage of the cortex explain the higher risk for developing MTSS with an increased BMI (Yang et al., 2014). Therefore, in individuals with a high BMI, the training may need to be modified to allow for a progressive increase in activity that allows adaptations in muscle and bone remodelling that decrease the likelihood of MTSS resulting from the increased stress throughout the kinetic chain.

\section{Previous vigorous physical activity}

420 Participants who developed MTSS performed less vigorous physical activity compared to the ones without MTSS. Our results also support that every MET-h per week of vigorous physical activity reduces the risk of MTSS by as much as $25 \%$. It may well be that vigorous physical activity resulted in adaptations in the tibia, arch-support foot muscles and passive soft tissues (such as plantar aponeurosis and ligaments within the arch). According to Wolff's Law, bone adapts to the forces it experiences (Frost, 1990), and it may be that the bone adaptations induced by regular vigorous physical activity were adequate to prevent MTSS during the running programme. Another potential cause of MTSS may have been an earlier onset of muscle fatigue of the foot arch-support muscles (Headlee et al., 2008; Milgrom et al., 2007) and/or creep by repetitive loading of arch-supporting soft tissues (Welk et al., 2015), which may lead to increased dynamic foot pronation during the landing phase of running. The latter is consistent with the observation that plantar intrinsic foot muscles have a minimal effect on the stiffness of the foot arch (Farris et al., 2019) and hence the stiffness of the arch must be largely attributed to passive soft tissues, such as the plantar aponeurosis and ligaments within the arch.

\section{Dynamic foot posture}

The dynamic foot pronation during running was a significant predictor for the incidence of MTSS. In line with our study, Becker et al. (2018) have shown that athletes with MTSS have more pronounced foot pronation during running, while static foot posture was not associated with MTSS. Therefore, it appears that dynamic foot posture is a more important predictor of the risk of MTSS than static foot posture. It is possible that increased dynamic foot pronation increases the strain on the tibia through greater peak soleus muscle activity. This may develop into overuse and MTSS if the increased strain exceeds the tibia bone structural capacity. It may be speculated that runners benefit from control of dynamic foot pronation using arch-support foot orthoses and thereby reduce the strain on the tibia. In line with this, a previous study suggested that clinical benefits of archsupport foot orthoses are related to altered magnitude, location and temporal pattern of ground reaction forces during gait (Dowling et al., 2014), but this requires further investigation.

\section{Previous history of MTSS}

Our data show that a previous history of MTSS was greater in the MTSS group (52\%) than in the healthy group (17\%) and participants who previously had MTSS were almost 4.67 times more likely to experience MTSS than those without a history of MTSS. Navy recruits (Garnock et al., 2018) and cross-country runners (Reinking et al., 2010) with an MTSS history, had, respectively, 18.28 and 20.09 times higher risk to develop MTSS again compared to those without a history of the condition. In people who suffer from MTSS, the repetitive force of running may prevent the affected tibia site from proper healing. In support of this, a previous study found residual evidence of bone demineralization for up to 8 years after an MTSS episode (Magnusson et al., 2003). Given that a history of MTSS is a nonmodifiable risk factor, the best strategy is to focus on the prevention of MTSS.

\section{EMG amplitude of soleus muscle}

Our study showed that before the start of the season, SO peak EMG amplitude was higher in people who developed MTSS than those who did not develop MTSS. This is in line with studies that reported an increased strain by a more active SO muscle on the tibia as a possible risk factor of MTSS (Beck \& Osternig, 1994; Brown, 2016; Stickley et al., 2009). The SO is attached to the medial calcaneus, and large and longer duration of traction forces associated with over-activity of the SO during supination in the propulsive period after over-pronation of the calcaneus upon landing (Brown, 2016; Hunt \& Smith, 2004) may result in severe strains to the distal third of the posteromedial tibia. If, without proper recovery, this is repeated over and over again during running it may ultimately lead to MTSS. The high dynamic foot pronation in MTSS participants during running may be related to a modified alignment of the rear-foot bones along with less stable foot articulations and an increase of the tibial internal rotation during the stance phase (Hunt \& Smith, 2004). In turn, altered rear-foot bone configuration changes the line of pull for the SO muscle, requiring a higher activity of SO muscle to produce the same torque. Considering that foot pronation increases with the fatigue of foot intrinsic muscles (Headlee et al., 2008), foot and arch exercises can improve the rigidity of the foot arch that helps to better control the foot pronation and tibial internal rotation, and therefore be useful in preventing and/or treating of MTSS. This is an area that needs more exploration.

\section{Limitations}

First, the participants of this study were university students and these results may not be applicable to other populations. We can also not exclude the role of possible training-related differences in the development of MTSS. This is unlikely to be a major problem in our study as the participants underwent a similar training programme. As has been suggested in a previous study (Winters et al., 2018), MTSS can be reliably diagnosed by assessing MTSS history and physical examination. However, imaging can be used to rule out other common causes of exerciseinduced leg pain. In a surface EMG, cross-talk from neighbouring muscles cannot be eliminated entirely. However, SENIAM recommendations were followed to minimize EMG cross-talk between the muscles. Therefore, we believe that the effect of cross-talk on the results in this study was negligible. We cannot exclude the role of increased flexor 
digitorum longus (Beck \& Osternig, 1994; Edama et al., 2017) and tibialis posterior (Saxena et al., 1990) activity that have been reported as risk factors for the development of MTSS. However, the measurement of the activity of these muscles is invasive and requires intramuscular EMG electrodes and are therefore not conducive for day to day practice.

\section{Conclusions}

This study is the first to identify that a high soleus, but not TA, peak EMG amplitude during the propulsion phase of running is a contributing risk factor for MTSS. More significant is the observation in this prospective study that participants who develop MTSS have a greater body mass, higher dynamic foot pronation, previous history of MTSS, and lower previous vigorous physical activity level. A model that includes BMI, peak amplitude of SO EMG, previous history of MTSS, DAI and physical activity demonstrates good classification accuracy of $88.6 \%$ of the participants who eventually developed MTSS. Preventive screening methods and programmes should consider broad preseason evaluations which assess these multiple factors to reduce the incidence of MTSS. The results of this study support that although peak soleus EMG is a predictor of the incidence of MTSS, its contribution is limited in comparison to the predictive power of BMl, dynamic foot index, previous history of MTSS and level of PA. Thus, screening for the latter parameters, without considering the soleus peak EMG, provides an easy and quick screening tool to predict the risk of MTSS.

\section{Acknowledgments}

The authors would like to thankall the university students who participated in this study and the CESR lab members at the Shahrood University of Technology as well for their assistance in data collection and data management.

\section{Disclosure statement}

The authors report no conflict of interest.

\section{ORCID}

Aynollah Naderi (iD) http://orcid.org/0000-0003-4765-8953

Maarten H. Moen (iD http://orcid.org/0000-0002-5980-4631

Hans Degens (iD) http://orcid.org/0000-0001-7399-4841

\section{References}

Alfayez, S. M., Ahmed, M. L., \& Alomar, A. Z. (2017). A review article of medial tibial stress syndrome. Journal of Musculoskeletal Surgery and Research, 1 (1), 2-5. https://doi.org/10.4103/jmsr.jmsr_13_17

Beck, B. R., \& Osternig, L. R. (1994). Medial tibial stress syndrome. The location of muscles in the leg in relation to symptoms. Journal of Bone and Joint Surgery, 76(7), 1057-1061. https://doi.org/10.2106/00004623199407000-00015

Becker, J., Nakajima, M., \& Wu, W. F. (2018). Factors contributing to medial tibial stress syndrome in runners: A prospective study. Medicine and Science in Sports and Exercise, 50(10), 2092-2100. https://doi.org/10. 1249/MSS.0000000000001674

570 Bouche, R. T., \& Johnson, C. H. (2007). Medial tibial stress syndrome (Tibial fasciitis): A proposed pathomechanical model involving fascial traction.
Journal of the American Podiatric Medical Association, 97(1), 31-36. https://doi.org/10.7547/0970031

Brown, A. A. (2016). Medial tibial stress syndrome: Muscles located at the site of pain. Scientifica, 2016(097489), 1-4. https://doi.org/10.1155/2016/7097489

Cavanagh, P. R., \& Rodgers, M. M. (1987). The arch index: A useful measure from footprints. Journal of Biomechanics, 20(5), 547-551. https://doi.org/ 10.1016/0021-9290(87)90255-7

Couture, C. J., \& Karlson, K. A. (2002). Tibial stress injuries: Decisive diagnosis and treatment of 'shin splints'. The Physician and Sportsmedicine, 30(6), 29-36. https://doi.org/10.3810/psm.2002.06.337

De Cock, A., Willems, T., Witvrouw, E., Vanrenterghem, J., \& De Clercq, D. (2006). A functional foot type classification with cluster analysis based on plantar pressure distribution during jogging. Gait \& Posture, 23(3), 339-347. https://doi.org/10.1016/j.gaitpost.2005.04.011

DeLacerda, F. G. (1980). The relationship of foot pronation, foot position, and electromyography of the anterior tibialis muscle in three subjects with different histories of shinsplints. Journal of Orthopaedic \& Sports Physical Therapy, 2(2), 60-64. https://doi.org/10.2519/jospt.1980.2.2.60

Dowling, G. J., Murley, G. S., Munteanu, S. E., Smith, M. M. F., Neal, B. S., Griffiths, I. B., \& Collins, N. J. (2014). Dynamic foot function as a risk factor for lower limb overuse injury: A systematic review. Journal of Foot and Ankle Research, 7(1), 53. https://doi.org/10.1186/s13047-014-0053-6

Edama, M., Onishi, H., Kubo, M., Takabayashi, T., Yokoyama, E., Inai, T., \& Kageyama, I. (2017). Gender differences of muscle and crural fascia origins in relation to the occurrence of medial tibial stress syndrome. Scandinavian Journal of Medicine \& Science in Sports, 27(2), 203-208. https://doi.org/10.1111/sms.12639

Edwards, P. H., Wright, M. L., \& Hartman, J. F. (2005). A practical approach for the differential diagnosis of chronic leg pain in the athlete. The American Journal of Sports Medicine, 33(8), 1241-1249. https://doi.org/10.1177/ 0363546505278305

Farris, D. J., Kelly, L. A., Cresswell, A. G., \& Lichtwark, G. A. (2019). The functional importance of human foot muscles for bipedal locomotion. Proceedings of the National Academy of Sciences, 116(5), 1645-1650. https://doi.org/10.1073/pnas.1812820116

Frost, H. M. (1990). Skeletal structural adaptations to mechanical usage (SATMU): 1. Redefining Wolff's law: The bone modeling problem. The Anatomical Record, 226(4), 403-413. https://doi.org/10.1002/ar. 1092260402

Garnock, C., Witchalls, J., \& Newman, P. (2018). Predicting individual risk for medial tibial stress syndrome in navy recruits. Journal of Science and Medicine in Sport, 21(6), 586-590. https://doi.org/10.1016/j.jsams.2017. 10.020

George, J. D., Vehrs, P. R., Allsen, P. E., Fellingham, G. W., \& Fisher, A. G. (1993). VO2max estimation from a submaximal 1-mile track jog for fit college-age individuals. Medicine and Science in Sports and Exercise, 25 (3), 401-406. https://doi.org/10.1249/00005768-199303000-00017

Headlee, D. L., Leonard, J. L., Hart, J. M., Ingersoll, C. D., \& Hertel, J. (2008). Fatigue of the plantar intrinsic foot muscles increases navicular drop. Journal of Electromyography and Kinesiology, 18(3), 420-425. https://doi. org/10.1016/j.jelekin.2006.11.004

Hunt, A. E., \& Smith, R. M. (2004). Mechanics and control of the flat versus normal foot during the stance phase of walking. Clinical Biomechanics, 19(4), 391-397. https://doi.org/10.1016/j.clinbiomech.2003.12.010

Kyröläinen, H., Avela, J., \& Komi, P. V. (2005). Changes in muscle activity with increasing running speed. Journal of Sports Sciences, 23(10), 1101-1109. https://doi.org/10.1080/02640410400021575

Lohman lii, E. B., Sackiriyas, K. S. B., \& Swen, R. W. (2011). A comparison of the spatiotemporal parameters, kinematics, and biomechanics between shod, unshod, and minimally supported running as compared to walking. Physical Therapy in Sport, 12(4), 151-163. https://doi.org/10. 1016/j.ptsp.2011.09.004

Lopes, A. D., Hespanhol, L. C., Yeung, S. S., \& Costa, L. O. P. (2012). What are the main running-related musculoskeletal injuries? Sports Medicine, 42 (10), 891-905. https://doi.org/10.2165/11631170-000000000-00000

Magnusson, H. I., Ahlborg, H. G., Karlsson, C., Nyquist, F., \& Karlsson, M. K. (2003). Low regional tibial bone density in athletes with medial tibial stress syndrome normalizes after recovery from symptoms. The American Journal of Sports Medicine, 31(4), 596-600. https://doi.org/10. $1177 / 03635465030310042001$

\section{5 \\ 580}

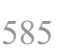


Milgrom, C., Radeva-Petrova, D. R., Finestone, A., Nyska, M., Mendelson, S., Benjuya, N., \& Burr, D. (2007). The effect of muscle fatigue on in vivo tibial strains. Journal of Biomechanics, 40(4), 845-850. https://doi.org/10.1080/ 02640410400021575

Moghaddam, M. B., Aghdam, F. B., Jafarabadi, M. A., Allahverdipour, H., Nikookheslat, S. D., \& Safarpour, S. (2012). The Iranian Version of International Physical Activity Questionnaire (IPAQ) in Iran: Content and construct validity, factor structure, internal consistency and stability. World Applied Sciences Journal, 18(8), 1073-1080. https://doi. org/10.5829/idosi.wasj.2012.18.08.754

Murley, G. S., Landorf, K. B., Menz, H. B., \& Bird, A. R. (2009). Effect of foot posture, foot orthoses and footwear on lower limb muscle activity during walking and running: A systematic review. Gait \& Posture, 29(2), 172-187. https://doi.org/10.1016/j.gaitpost.2008.08.015

Naderi, A., Baloochi, R., Dashti Rostami, K., Fourchet, F., \& Degens, H. (In press). Obesity, and foot muscle strength are associated with high dynamic plantar pressure during running. The Foot. https://doi.org/10. 1016/j.foot.2020.101683

Naderi, A., Degens, H., \& Sakinepoor, A. (2019). Arch-support foot-orthoses normalize dynamic in-shoe foot pressure distribution in medial tibial stress syndrome. European Journal of Sport Science, 19(2), 247-257. https://doi.org/10.1080/17461391.2018.1503337

Newman, P., Witchalls, J., Waddington, G., \& Adams, R. (2013). Risk factors associated with medial tibial stress syndrome in runners: $A$ systematic review and meta-analysis. Open Access Journal of Sports Medicine, 4(p), 229. https://doi.org/10.2147/OAJSM.S39331

Redmond, A. C., Crosbie, J., \& Ouvrier, R. A. (2006). Development and validation of a novel rating system for scoring standing foot posture: The Foot Posture Index. Clinical Biomechanics, 21(1), 89-98. https://doi. org/10.1016/j.clinbiomech.2005.08.002

Reinking, M. F., Austin, T. M., \& Hayes, A. M. (2010). Risk factors for self-reported exercise-related leg pain in high school cross-country athletes. Journal of Athletic Training, 45(1), 51-57. https://doi.org/10. 4085/1062-6050-45.1.51

Saxena, A., O'Brien, T., \& Bunce, D. (1990). Anatomic dissection of the tibialis posterior muscle and its correlation to medial tibial stress syndrome. The Journal of Foot Surgery, 29(2), 105-108.
Scott, L. A., Murley, G. S., \& Wickham, J. B. (2012). The influence of footwear on the electromyographic activity of selected lower limb muscles during walking. Journal of Electromyography and Kinesiology, 22(6), 1010-1016. https://doi.org/10.1016/j.jelekin.2012. 06.008

Stickley, C. D., Hetzler, R. K., Kimura, I. F., \& Lozanoff, S. (2009). Crural fascia and muscle origins related to medial tibial stress syndrome symptom location. Medicine and Science in Sports and Exercise, 41(11), 1991-1996. https://doi.org/10.1249/MSS.0b013e3181a6519c

Tweed, J. L., Avil, S. J., Campbell, J. A., \& Barnes, M. R. (2008). Etiologic factors in the development of medial tibial stress syndrome: A review of the literature. Journal of the American Podiatric Medical Association, 98(2), 107-111. https://doi.org/10.7547/0980436

Welk, A. B., Haun, D. W., Clark, T. B., \& Kettner, N. W. (2015). Use of high-resolution ultrasound to measure changes in plantar fascia thickness resulting from tissue creep in runners and walkers. Journal of Manipulative and Physiological Therapeutics, 38(1), 81-85. https://doi. org/10.1016/j.jmpt.2014.10.008

Winters, M., Bakker, E., Moen, M., Barten, C., Teeuwen, R., \& Weir, A. (2018). Medial tibial stress syndrome can be diagnosed reliably using history and physical examination. British Journal of Sports Medicine, 52(19), 1267-1272. https://doi.org/10.1136/bjsports-2016097037

Winters, M., Bon, P., Bijvoet, S., Bakker, E. W., \& Moen, M. H. (2016). Are ultrasonographic findings like periosteal and tendinous edema associated with medial tibial stress syndrome? A case-control study. Journal of Science and Medicine in Sport, 20(2), 128-133. https://doi. org/10.1016/j.jsams.2016.07.001

Yang, P.-F., Sanno, M., Ganse, B., Koy, T., Brüggemann, G.-P., Müller, L. P., \& Rittweger, J. (2014). Torsion and antero-posterior bending in the in vivo human tibia loading regimes during walking and running. PloS One, 9(4), e94525. https://doi.org/10.1371/journal. pone.0094525

Yates, B., \& White, S. (2004). The incidence and risk factors in the development of medial tibial stress syndrome among naval recruits. The American Journal of Sports Medicine, 32(3), 772-780. https://doi.org/10. $1177 / 0095399703258776$ 